\title{
ESTIMATION OF ABILITY WITH REDUCED ASYMPTOTIC MEAN SQUARE ERROR IN ITEM RESPONSE THEORY
}

\author{
Haruhiko Ogasawara*
}

\begin{abstract}
A method of the weighted score or penalized likelihood for estimation of ability reducing the asymptotic mean square error is derived. In this method, associated item parameters are assumed to be given or estimated by using a separate calibration sample with the size of an appropriate order. The method can be seen as an extension of the weighted likelihood method that removes the asymptotic bias of the maximum likelihood estimator. In the proposed method, some bias is retained while variance is reduced by using a multiplicative constant for the weight in the weighted score. A lower bound of the constant minimizing the asymptotic mean square error is found under the logistic model having identical items. The lower bound is numerically also shown to be reasonable in the case of the 3-parameter logistic model, with and without model misspecification.
\end{abstract}

Key words and phrases: 3-parameter logistic model, ability estimation, asymptotic bias, Bayes modal, higher-order asymptotic variance, mean square error, weighted score.

\section{Introduction}

There are many methods for estimation of ability or the person parameter in item response theory (IRT). The maximum likelihood estimator (MLE; Lord (1953), Birnbaum (1968), Section 20.3), when item parameters are given, or seen as fixed, is a standard estimator. The maximum a posteriori or Bayes modal estimator (BME; Samejima (1969), Chapter 2; Bock and Aitkin (1981)) that typically uses the informative standard normal prior is also available. Warm (1989) derived the so-called weighted likelihood (WL) method that gives the ability estimator without the asymptotic bias of order $O\left(n^{-1}\right)$, with $n$ being the number of items, under the assumption that the IRT model is correctly specified. The WL method can be seen as a special case of the weighted score or penalized likelihood method, removing the asymptotic bias (Firth (1993)).

The WL method can be used for the familiar 3-parameter logistic model (3PLM). When the 2-parameter logistic model (2PLM) holds, the estimator derived by the WL method (WLE) becomes identical to the BME, using the Jeffreys (1946; 1961, Section 3.10) non-informative prior. In this paper, the latter is called the Jeffreys modal estimator (JME), while the BME refers to the estimator using the standard normal prior. It is known that the JME under canonical parametrization in the exponential family has no asymptotic bias of

\footnotetext{
Received September 2, 2013. Revised November 27, 2013. Accepted December 31, 2013.

*Department of Information and Management Science, Otaru University of Commerce, 3-5-21, Midori, Otaru 047-8501 Japan.
} 
order $O\left(n^{-1}\right)$ (Firth (1993)), where $n$ is seen as a general sample size e.g., the number of items in the case of IRT. The ability parameter in the 2PLM belongs to this family, while that of the 3PLM does not.

Ogasawara (2013a, Equation (2.4)) dealt with the ML, BM, JM and WL methods as special cases of the weighted score method with the general weight $g(\theta)$, a differentiable function of $\theta$. Ogasawara (2013a), then derived the asymptotic cumulants up to the fourth order and the higher-order asymptotic variance of the estimator with $g(\theta)$ under possible model misspecification (p.m.m.), which gave the asymptotic mean square error $\left(\mathrm{MSE}_{O\left(n^{-2}\right)}\right)$ of the ability estimator up to order $O\left(n^{-2}\right)$. Ogasawara (2013b) derived a general method of bias adjustment that minimizes the $\mathrm{MSE}_{O\left(n^{-2}\right)}$, as an extension of $g(\theta)$ that removes the asymptotic bias of order $O\left(n^{-2}\right)$ under correct model specification (c.m.s.). Ogasawara (2013b) uses the multiplicative constant $k_{\min }$ for $k g(\theta)$ minimizing the $\mathrm{MSE}_{O\left(n^{-2}\right)}$ among this family. Note that $k$ corresponds to the precision parameter or the reciprocal of variance in the case of Bayesian estimation with the normal prior. Unfortunately, the constant $k_{\text {min }}$ generally includes the unknown parameter $\theta$. In this paper, a fixed lower bound of $k_{\min }$ is derived in the case of ability estimation under the logistic model having equivalent items without guessing parameters (LME; Birnbaum (1969), p. 260). Numerical illustration is given using the 3PLM with and without model misspecification, where the lower bound for the LME is numerically shown to be reasonable even for the 3PLM.

\section{Preliminary properties}

Under the 3PLM, write

$$
\begin{aligned}
& P_{m} \equiv \operatorname{Pr}\left(U_{m}=1 \mid \theta, a_{m}, b_{m}, c_{m}\right)=c_{m}+\frac{1-c_{m}}{1+\exp \left\{-D a_{m}\left(\theta-b_{m}\right)\right\}}, \\
& Q_{m} \equiv 1-P_{m} \quad(m=1, \ldots, n),
\end{aligned}
$$

where $U_{m}$ is the dichotomous variable with $U_{m}=1$ and 0 for correct and incorrect responses, respectively by an examinee with $\theta ; D=1.7$; and $a_{m}, b_{m}$ and $c_{m}$ are item parameters. Note that the LME without guessing parameters gives $P_{m}=1 /[1+\exp \{-D a(\theta-b)\}]$ with $a_{m}=a, b_{m}=b$ and $c_{m}=0(m=1, \ldots, n)$. When the model is misspecified, define

$$
P_{\mathrm{T} m} \equiv E_{\mathrm{T}}\left(U_{m} \mid \theta\right), \quad Q_{\mathrm{T} m} \equiv 1-P_{\mathrm{T} m} \quad(m=1, \ldots, n),
$$

where $E_{\mathrm{T}}(\cdot)$ indicates that the expectation is taken using the true distribution of $U_{m}$ rather than the 3PLM for at least one item.

Let $\gamma=\left(a_{1}, b_{1}, c_{1}, \ldots, a_{n}, b_{n}, c_{n}\right)^{\prime}$. In practice, $\gamma$ is unknown and estimated by the responses of examinees with the sample size $N$ for item calibration. An examinee with the population ability $\theta_{0}$, to be estimated, is assumed to be separate from the calibration sample, which is a typical situation encountered in practice, after the associated test is developed with the estimate $\hat{\gamma}$ being fixed for estimation of the ability. Even in this situation, $\hat{\gamma}$ is subject to sampling 
variation. However, Ogasawara (2013c) derived that the estimators e.g., MLE of $\theta_{0}$ using $\hat{\gamma}$ under $N=O\left(n^{5 / 2}\right)$ give the same asymptotic cumulants up to the fourth order and the higher-order asymptotic variance as those using $\gamma$. This result gives the same $\mathrm{MSE}_{O\left(n^{-2}\right)}$ for the estimator using $\hat{\gamma}$ as that derived by $\gamma$. In this paper, $N=O\left(n^{5 / 2}\right)$ is assumed, where $\gamma$ is used in place of $\hat{\gamma}$ for simplicity of notation. That is, though the results look as if derived when $\gamma$ is known, they hold also when using $\hat{\gamma}$ under the assumption $N=O\left(n^{5 / 2}\right)$.

Let $L$ be the likelihood of $\theta$, when $\gamma$ and observed $\boldsymbol{u}=\left(u_{1}, \ldots, u_{n}\right)^{\prime}$ of dichotomous variables $U_{m}(m=1, \ldots, n)$ are given. Then, $L=L(\theta \mid \gamma, \boldsymbol{u})=$ $\prod_{m=1}^{n} P_{m}^{u_{m}} Q_{m}^{1-u_{m}}$. Define $\bar{l}_{\mathrm{GW}}$ as

$$
\frac{\partial \bar{l}_{\mathrm{GW}}}{\partial \theta} \equiv \frac{\partial n^{-1} \log L}{\partial \theta}+n^{-1} g(\theta) \equiv \frac{\partial \bar{l}}{\partial \theta}+n^{-1} g(\theta),
$$

where $g(\theta)$ is the general weight in the weighted score method mentioned earlier. A special case of the weight is the log prior derivative in Bayesian estimation. The weight becomes

$$
\begin{array}{llll}
g(\theta) & =0 \quad \text { for } \mathrm{ML} \quad g(\theta)=-\theta & & \text { for BM, } \\
g(\theta) & =\bar{i}^{\prime} /(2 \bar{i}) \quad \text { for } \mathrm{JM} \text { and } & g(\theta)=\bar{j} /(2 \bar{i}) & \text { for } \mathrm{WL}
\end{array}
$$

where $\bar{i}=O(1)$ is the test or Fisher information averaged over items i.e., $\bar{i} \equiv$ $n^{-1} \sum_{m=1}^{n} P_{m}^{\prime 2} /\left(P_{m} Q_{m}\right)$, where $P_{m}^{\prime}=\partial P_{m} / \partial \theta$; and $\bar{j} \equiv n^{-1} \sum_{m=1}^{n} P_{m}^{\prime} P_{m}^{\prime \prime} /$ $\left(P_{m} Q_{m}\right)$ with $P_{m}^{\prime \prime}=\partial^{2} P_{m} / \partial \theta^{2}$ and $P_{m}^{\prime \prime \prime}=\partial^{3} P_{m} / \partial \theta^{3}$ for later use. The estimator $\hat{\theta}_{\mathrm{GW}}$ is defined as that maximizing $\bar{l}_{\mathrm{GW}}$. That is, $\hat{\theta}_{\mathrm{GW}}$ includes $\hat{\theta}_{\mathrm{ML}}, \hat{\theta}_{\mathrm{BM}}$, $\hat{\theta}_{\mathrm{JM}}$ and $\hat{\theta}_{\mathrm{WL}}$, which are the estimators using $g(\theta)$ for ML, BM, JM and WL, respectively.

The population value $\theta_{0}$ under model misspecification (m.m.) is defined as a solution maximizing $\bar{l}$, where observations $u_{m}(m=1, \ldots, n)$ are replaced by $P_{\mathrm{T} m}(m=1, \ldots, n)($ see $(2.2))$. Let

$$
\begin{aligned}
E_{\mathrm{T}}\left(\hat{\theta}_{\mathrm{GW}}-\theta_{0}\right) \equiv & n^{-1} \alpha_{\mathrm{GW} 1}+O\left(n^{-2}\right) \\
& =n^{-1}\left\{\alpha_{\mathrm{ML} 1}-\lambda^{-1} g\left(\theta_{0}\right)\right\}+O\left(n^{-2}\right), \\
E_{\mathrm{T}}\left\{\left(\hat{\theta}_{\mathrm{GW}}-\theta_{0}\right)^{2}\right\} \equiv & n^{-1} \alpha_{\mathrm{GW} 2}+n^{-2} \alpha_{\mathrm{GW} \Delta 2}+O\left(n^{-3}\right) \\
& =n^{-1} \alpha_{\mathrm{ML} 2}+n^{-2} \alpha_{\mathrm{GW} \Delta 2}+O\left(n^{-3}\right),
\end{aligned}
$$

where $n^{-1} \alpha_{\mathrm{GW} 1}$ is the asymptotic bias of order $O\left(n^{-1}\right)$, which is equal to $n^{-1}\left\{\alpha_{\mathrm{ML} 1}-\lambda^{-1} g\left(\theta_{0}\right)\right\}$ (Ogasawara (2013a), Equation (3.9)), where $\alpha_{\mathrm{ML} 1}$ is $\alpha_{\mathrm{GW} 1}$ by ML and $\lambda \equiv E_{\mathrm{T}}\left(\partial^{2} \bar{l} /\left.\partial \theta^{2}\right|_{\theta=\theta_{0}}\right)$, which becomes the negative of the population averaged information denoted by $\bar{i}_{0}$ under c.m.s. In $(2.5), n^{-1} \alpha_{\mathrm{GW} 2}$ is the usual asymptotic variance of $\hat{\theta}_{\mathrm{GW}}$, which is equal to $n^{-1} \alpha_{\mathrm{ML} 2}$ given by $\hat{\theta}_{\mathrm{ML}}$ (Ogasawara (2013a), Equation (3.9)). That is, $n^{-1} \alpha_{\mathrm{ML} 2}$ is unchanged irrespective of $g(\theta)$. The quantity $n^{-2} \alpha_{\mathrm{GW} \Delta 2}$ is the added higher-order asymptotic variance of $\hat{\theta}_{\mathrm{GW}}$, which generally depends on $g(\theta)$. 
Under p.m.m., Ogasawara (2013a, Theorem 1) gave

$$
\begin{aligned}
& \operatorname{MSE}_{O\left(n^{-2}\right)}\left(\hat{\theta}_{\mathrm{GW}}\right) \\
& \begin{array}{l}
=n^{-1} \alpha_{\mathrm{ML} 2} \\
\quad+n^{-2}\left[\alpha_{\mathrm{ML} 22}+\alpha_{\mathrm{ML} 1}^{2}-2 \lambda^{-1} g^{\prime}\left(\theta_{0}\right) \alpha_{\mathrm{ML} 2}\right. \\
\quad-2 g\left(\theta_{0}\right)\left\{\lambda^{-3} E_{\mathrm{T}}\left(n\left(\partial \bar{l} /\left.\partial \theta\right|_{\theta=\theta_{0}}\right)\left(\partial^{2} \bar{l} /\left.\partial^{2} \theta\right|_{\theta=\theta_{0}}-\lambda\right)\right)\right. \\
\left.\left.\quad-\lambda^{-2} E_{\mathrm{T}}\left(\partial^{3} \bar{l} /\left.\partial^{3} \theta\right|_{\theta=\theta_{0}}\right) \alpha_{\mathrm{ML} 2}+\lambda^{-1} \alpha_{\mathrm{ML} 1}\right\}+\lambda^{-2} g\left(\theta_{0}\right)^{2}\right],
\end{array}
\end{aligned}
$$

where $\alpha_{\mathrm{ML} \Delta 2}$ is $\alpha_{\mathrm{GW} \Delta 2}$ by ML and $g^{\prime}\left(\theta_{0}\right)=\partial g(\theta) /\left.\partial \theta\right|_{\theta=\theta_{0}}$. Under c.m.s. using $\alpha_{\mathrm{ML1}}=-\bar{j}_{0} /\left(2 \bar{i}_{0}^{2}\right)$ (Lord (1983), Warm (1989)) and the Bartlett identity, (2.6) becomes

$$
\begin{aligned}
\operatorname{MSE}_{O\left(n^{-2}\right)}\left(\hat{\theta}_{\mathrm{GW}}\right)= & n^{-1} \bar{i}_{0}^{-1}+n^{-2}\left\{\alpha_{\mathrm{ML} \Delta 2}+\alpha_{\mathrm{ML} 1}^{2}+2 \bar{i}_{0}^{-2} g^{\prime}\left(\theta_{0}\right)\right. \\
& \left.-\bar{i}_{0}^{-3}\left(2 \bar{i}_{0}^{\prime}+\bar{j}_{0}\right) g\left(\theta_{0}\right)+\bar{i}_{0}^{-2} g\left(\theta_{0}\right)^{2}\right\}
\end{aligned}
$$

(Ogasawara (2013a), Equation (3.14)), where $\bar{j}_{0}$ is the population counterpart of $\bar{j}$. Note that under p.m.m.,

$$
\operatorname{MSE}_{O\left(n^{-2}\right)}\left(\hat{\theta}_{\mathrm{ML}}\right)=n^{-1} \alpha_{\mathrm{ML} 2}+n^{-2}\left(\alpha_{\mathrm{ML} \Delta 2}+\alpha_{\mathrm{ML} 1}^{2}\right),
$$

while under c.m.s.,

$$
\operatorname{MSE}_{O\left(n^{-2}\right)}\left(\hat{\theta}_{\mathrm{ML}}\right)=n^{-1} \bar{i}_{0}^{-1}+n^{-2}\left(\alpha_{\mathrm{ML} \Delta 2}+\alpha_{\mathrm{ML} 1}^{2}\right),
$$

where $\alpha_{\mathrm{ML} \Delta 2}$ and $\alpha_{\mathrm{ML} 1}$ in (2.8) are generally different from those in (2.9), respectively (see Ogasawara (2012), Equation (3.5); and Ogasawara (2013a), Equation (3.4) and Appendix A.2). In (2.6) and (2.7), $\mathrm{MSE}_{O\left(n^{-1}\right)}\left(\hat{\theta}_{\mathrm{GW}}\right)$ s of order $O\left(n^{-1}\right)$ are given by $n^{-1} \alpha_{\mathrm{ML} 2}$ and $n^{-1} \bar{i}_{0}^{-1}$, respectively, where the asymptotic bias up to order $O\left(n^{-1 / 2}\right)$ is zero.

\section{Main results}

Main results are given from (2.7) under c.m.s. Multiply $g(\theta)$ by a constant $k$, whose $\hat{\theta}_{\mathrm{GW}}$ is denoted by $\hat{\theta}_{\mathrm{GW}(k)}$. Then, from $(2.7)$, we have

$$
\begin{aligned}
& \operatorname{MSE}_{O\left(n^{-2}\right)}\left(\hat{\theta}_{\mathrm{GW}(k)}\right) \\
& =n^{-1} \bar{i}_{0}^{-1}+n^{-2}\left[\alpha_{\mathrm{ML} \Delta 2}+\alpha_{\mathrm{ML} 1}^{2}\right. \\
& \left.\quad+k\left\{2 \bar{i}_{0}^{-2} g^{\prime}\left(\theta_{0}\right)-\bar{i}_{0}^{-3}\left(2 \bar{i}_{0}^{\prime}+\bar{j}_{0}\right) g\left(\theta_{0}\right)\right\}+k^{2} \bar{i}_{0}^{-2} g\left(\theta_{0}\right)^{2}\right]
\end{aligned}
$$

which is minimized when $k$ is

$$
k_{\min }=-g\left(\theta_{0}\right)^{-2} g^{\prime}\left(\theta_{0}\right)+\bar{i}_{0}^{-1}\left\{\bar{i}_{0}^{\prime}+\left(\bar{j}_{0} / 2\right)\right\} g\left(\theta_{0}\right)^{-1},
$$

where $g\left(\theta_{0}\right) \neq 0$ is assumed. Define $\hat{\theta}_{\mathrm{WL}(k)}$ as $\hat{\theta}_{\mathrm{GW}(k)}$ when $k g(\theta)=k \bar{j} /(2 \bar{i})$ (note $\hat{\theta}_{\mathrm{WL}(1)}=\hat{\theta}_{\mathrm{WL}} ; \hat{\theta}_{\mathrm{GW}(k)}$ will also be written as $\hat{\theta}_{\mathrm{GW}}$ when $k g(\theta)$ is seen as a new $g(\theta))$. That is, bias adjustment of the ability estimator is considered to be a 
special case of the use of $k g(\theta)$ (a similar adjustment by BM will be addressed later). Then, from $g\left(\theta_{0}\right)=\bar{j}_{0} /\left(2 \bar{i}_{0}\right)(3.2)$ becomes

$$
\begin{aligned}
k_{\min } & =-\frac{4 \bar{i}_{0}^{2}}{\bar{j}_{0}^{2}}\left(\frac{\bar{j}_{0}^{\prime}}{2 \bar{i}}-\frac{\bar{j}_{0} \bar{i}_{0}^{\prime}}{2 \bar{i}_{0}^{2}}\right)+\bar{i}_{0}^{-1}\left(\bar{i}_{0}^{\prime}+\frac{\bar{j}_{0}}{2}\right) \frac{2 \bar{i}_{0}}{\bar{j}_{0}} \\
& =-2 \bar{i}_{0} \bar{j}_{0}^{-2} \bar{j}_{0}^{\prime}+4 \bar{j}_{0}^{-1} \bar{i}_{0}^{\prime}+1
\end{aligned}
$$

where $\bar{j}_{0}^{\prime}=\partial \bar{j} /\left.\partial \theta\right|_{\theta=\theta_{0}}$.

As a special case of the 3PLM or 2PLM, consider the LME addressed earlier i.e., $a_{m}=a, b_{m}=b, c_{m}=0, P_{m}=P, Q_{m}=Q, P_{m}^{\prime}=P^{\prime}, P_{m}^{\prime \prime}=P^{\prime \prime}(m=$ $1, \ldots, n)$. This reduces to the binomial error model (Lord and Novick (1968), Chapter 23; Birnbaum (1969)). Then, we have the following theorem.

THEOREM 1. When the LME holds with the assumptions $\bar{j}_{0} \neq 0$ and $\theta_{0}$ being finite, a lower bound of $k_{\min }$ for $\hat{\theta}_{\mathrm{WL}(k)}$ independent of $\theta_{0}$ is 3 , which gives the $\operatorname{MSE}_{O\left(n^{-2}\right)}\left(\hat{\theta}_{\mathrm{WL}(3)}\right)$ smaller than that given by $0 \leq k<3$.

Proof. Under the LME, $P^{\prime}=D a P Q$ gives

$$
\begin{aligned}
& \bar{i}_{0}=P^{\prime 2} /(P Q)=D^{2} a^{2} P Q, \\
& \bar{j}_{0}=\bar{i}_{0}^{\prime}=P^{\prime} P^{\prime \prime} /(P Q)=D a P^{\prime \prime}=D^{3} a^{3}(1-2 P) P Q, \quad \text { and } \\
& \bar{j}_{0}^{\prime}=D^{4} a^{4}\left(1-6 P+6 P^{2}\right) P Q .
\end{aligned}
$$

From (3.4), (3.3) becomes

$$
\begin{aligned}
k_{\min } & =-2 \frac{\left(D^{2} a^{2} P Q\right)\left\{D^{4} a^{4}\left(1-6 P+6 P^{2}\right) P Q\right\}}{\left\{D^{3} a^{3}(1-2 P) P Q\right\}^{2}}+5 \\
& =-2 \frac{1-6 P+6 P^{2}}{(1-2 P)^{2}}+5=-2 \frac{(1-2 P)^{2}-2 P(1-P)}{(1-2 P)^{2}}+5 \\
& =3+\frac{4 P(1-P)}{(1-2 P)^{2}} \\
& >3,
\end{aligned}
$$

where the denominator $\left\{D^{3} a^{3}(1-2 P) P Q\right\}^{2}=\bar{j}_{0}^{2} \neq 0$ by assumption; the strict inequality is owing to the assumption of finite $\theta_{0}$. Since $\operatorname{MSE}_{O\left(n^{-2}\right)}\left(\hat{\theta}_{\mathrm{WL}(k)}\right)$ is a quadratic function of $k$ with the coefficient of $k^{2}$ being $\bar{i}_{0}^{-2} g\left(\theta_{0}\right)^{2}=\bar{j}_{0}^{2} /\left(4 \bar{i}_{0}^{4}\right)>0$ by assumption, $\operatorname{MSE}_{O\left(n^{-2}\right)}\left(\hat{\theta}_{\mathrm{WL}(3)}\right)$ is smaller than $\operatorname{MSE}_{O\left(n^{-2}\right)}\left(\hat{\theta}_{\mathrm{WL}(k)}\right)$ for $0 \leq$ $k<3$.

From Theorem 1, we immediately have

COROLLARY 1. Under the LME with the assumptions as in Theorem 1,

$$
\operatorname{MSE}_{O\left(n^{-2}\right)}\left(\hat{\theta}_{\mathrm{WL}(3)}\right)<\operatorname{MSE}_{O\left(n^{-2}\right)}\left(\hat{\theta}_{\mathrm{WL}}\right)<\operatorname{MSE}_{O\left(n^{-2}\right)}\left(\hat{\theta}_{\mathrm{ML}}\right) .
$$


Corollary 1 suggests an excellent asymptotic property of Warm's WLE or $\hat{\theta}_{\mathrm{WL}}$ in that not only its asymptotic bias of order $O\left(n^{-1}\right)$ is zero, but also the $\mathrm{MSE}_{O\left(n^{-2}\right)}$ is smaller than that by ML in this model. Note, however, that as Firth (1993, p. 36) stated "It is not an assumption of this work that bias reduction is always desirable", $\hat{\theta}_{\mathrm{WL}}$ is not always the "best" one among the estimators $\hat{\theta}_{\mathrm{WL}(k)}$. Since the MSE is an overall index of risk considering both variance and bias, $\hat{\theta}_{\mathrm{WL}(3)}$ or $\hat{\theta}_{\mathrm{WL}(k)}$ with $1<k<3$ may be more reasonable than $\hat{\theta}_{\mathrm{WL}}$. So far, being unbiased seems to have been overemphasized (implicitly) depreciating small variance. This is due partially to the relatively tractable property of the asymptotic bias of order $O\left(n^{-1}\right)$ rather than the higher-order asymptotic variance up to order $O\left(n^{-2}\right)$. Note that the former is usually given by the asymptotic expansion of an estimator up to order $O_{p}\left(n^{-1}\right)$ whereas the latter is given by that up to order $O_{p}\left(n^{-3 / 2}\right)$.

In ability tests using IRT, the 2PLM and 3PLM are more common than the LME. Under the 2PLM, we have

$$
\begin{aligned}
\bar{i}_{0} & =n^{-1} \sum_{m=1}^{n} P_{m}^{\prime 2} /\left(P_{m} Q_{m}\right)=n^{-1} D^{2} \sum_{m=1}^{n} a_{m}^{2} P_{m} Q_{m} \\
\bar{j}_{0} & =\bar{i}_{0}^{\prime}=n^{-1} \sum_{m=1}^{n} P_{m}^{\prime} P_{m}^{\prime \prime} /\left(P_{m} Q_{m}\right) \\
& =n^{-1} D \sum_{m=1}^{n} a_{m} P_{m}^{\prime \prime}=n^{-1} D^{3} \sum_{m=1}^{n} a_{m}^{3}\left(1-2 P_{m}\right) P_{m} Q_{m}, \quad \text { and } \\
\bar{j}_{0}^{\prime} & =n^{-1} D^{4} \sum_{m=1}^{n} a_{m}^{4}\left(1-6 P_{m}+6 P_{m}^{2}\right) P_{m} Q_{m} .
\end{aligned}
$$

From (3.7), (3.3) becomes

$$
\begin{aligned}
k_{\min }= & -2 \frac{\left(\sum_{m=1}^{n} a_{m}^{2} P_{m} Q_{m}\right)\left\{\sum_{m=1}^{n} a_{m}^{4}\left(1-6 P_{m}+6 P_{m}^{2}\right) P_{m} Q_{m}\right\}}{\left\{\sum_{m=1}^{n} a_{m}^{3}\left(1-2 P_{m}\right) P_{m} Q_{m}\right\}^{2}}+5 \\
= & -2 \frac{\sum_{m=1}^{n} a_{m}^{2} P_{m} Q_{m}}{\left\{\sum_{m=1}^{n} a_{m}^{3}\left(1-2 P_{m}\right) P_{m} Q_{m}\right\}^{2}} \\
& \times \sum_{m=1}^{n}\left\{a_{m}^{4}\left(1-2 P_{m}\right)^{2}-2 a_{m}^{4} P_{m}\left(1-P_{m}\right)\right\} P_{m} Q_{m}+5 \\
= & -2 \frac{\left(\sum_{m=1}^{n} a_{m}^{2} P_{m} Q_{m}\right)\left\{\sum_{m=1}^{n} a_{m}^{4}\left(1-2 P_{m}\right)^{2} P_{m} Q_{m}\right\}}{\left\{\sum_{m=1}^{n} a_{m}^{3}\left(1-2 P_{m}\right) P_{m} Q_{m}\right\}^{2}} \\
& +4 \frac{\left(\sum_{m=1}^{n} a_{m}^{2} P_{m} Q_{m}\right)\left\{\sum_{m=1}^{n} a_{m}^{4}\left(P_{m} Q_{m}\right)^{2}\right\}}{\left\{\sum_{m=1}^{n} a_{m}^{3}\left(1-2 P_{m}\right) P_{m} Q_{m}\right\}^{2}}+5 .
\end{aligned}
$$

In (3.8), the first term on the right-hand side of the last equality is smaller than or equal to -2 by the Cauchy-Schwarz inequality (the equality holds if the LME holds) while the second term is positive. It is difficult to find a reasonable positive lower bound independent of $\theta_{0}$ as in Theorem 1 .

Under the 3PLM, $k_{\min }$ given by $(3.3)$ becomes

$$
k_{\min }=-2 \bar{i}_{0} \bar{j}_{0}^{-2} \bar{j}_{0}^{\prime}+4 \bar{j}_{0}^{-1} \bar{i}_{0}^{\prime}+1
$$




$$
\begin{aligned}
= & -2 \frac{\sum_{m=1}^{n} P_{m}^{\prime 2} /\left(P_{m} Q_{m}\right)}{\left\{\sum_{m=1}^{n} P_{m}^{\prime} P_{m}^{\prime \prime} /\left(P_{m} Q_{m}\right)\right\}^{2}} \\
& \times \sum_{m=1}^{n}\left\{\frac{P_{m}^{\prime} P_{m}^{\prime \prime \prime}+P_{m}^{\prime \prime 2}}{P_{m} Q_{m}}-\frac{P_{m}^{\prime 2} P_{m}^{\prime \prime}\left(1-2 P_{m}\right)}{\left(P_{m} Q_{m}\right)^{2}}\right\} \\
& +4 \frac{\sum_{m=1}^{n}\left\{2 P_{m}^{\prime} P_{m}^{\prime \prime} /\left(P_{m} Q_{m}\right)-P_{m}^{\prime 3}\left(1-2 P_{m}\right) /\left(P_{m} Q_{m}\right)^{2}\right\}}{\sum_{m=1}^{n} P_{m}^{\prime} P_{m}^{\prime \prime} /\left(P_{m} Q_{m}\right)}+1
\end{aligned}
$$

Since the 2PLM and 3PLM are similar to the LME in a crude sense, the results of Theorem 1 and consequently Corollary 1 are expected to be partially conveyed to the 2PLM and 3PLM. This is numerically illustrated in the next section for the 3PLM that is probably more often used than the 2PLM in current ability tests.

\section{Numerical illustration for the 3PLM}

Numerical illustration is given using the artificial population item parameters of the 3PLM given by the method of Ogasawara (2012, 2013a), which mimics typical values found in practice (e.g., Lord (1975), Figures 1-4; Kolen and Brennan (2004), Table 6.5). That is, for $a$-, $b$ - and $c$-parameters, the independent normal distributions $a_{m} \sim N\left(0.8,0.3^{2}\right), b_{m} \sim N\left(0.5,1^{2}\right)$ and $c_{m} \sim N\left(0.15,0.07^{2}\right)$ $(m=1, \ldots, n)$ are used, where the values $0.4 \leq a_{m} \leq 1.7,-1.5 \leq b_{m} \leq 2.2$ and $0.01 \leq c_{m} \leq 0.29$ are employed. Sample sizes $n=50,100$ and 300 are used under c.m.s. On the other hand, $n=100$ is used under m.m. $P_{\mathrm{T} m}(m=1, \ldots, n)$ under m.m. are generated by perturbing the term $-D a_{m}\left(\theta-b_{m}\right)$ such that $-D a_{m}\left(\theta-b_{m}\right)+e_{m}+e^{*}$ with $e_{m} \sim N\left(0, D^{2} \sigma_{e}^{2}\right)$ and $\sigma_{e}^{2}=1$. An additional term $e^{*}$, which depends on $e_{m}(m=1, \ldots, n)$, is employed in order to have $E_{\mathrm{T}}\left(\partial \bar{l} /\left.\partial \theta\right|_{\theta=\theta_{0}}\right)=0\left(E_{\mathrm{T}}\left(\partial \bar{l}_{\mathrm{GW}} /\left.\partial \theta\right|_{\theta=\theta_{0}}\right)=0\right.$ with $\left.g\left(\theta_{0}\right)=0\right)$. The degree of misspecification is somewhat large in that the correlations between $P_{\mathrm{T} m}$ and $P_{m}$ over items are $0.567,0.574,0.535$ and 0.458 , when $\theta=-1,0,1$ and 2 , respectively. The simulated data when $\theta$ is equal to or smaller than -2 and when $\theta$ is greater than 2 are not used, since the data give unstable estimates of abilities.

The methods of ML, BM, JM, WL and WL $(k)$ with $k=2,3,4$ and $k_{\text {min }}$ are used, where $k_{\text {min }}$ under the 3PLM given by (3.9) is used for comparison though $k_{\text {min }}$ depends on $\theta_{0}$ that is unknown in practice. Table 1 shows the values of $k_{\text {min }}$ when $\theta=-3(1) 3$. Note that $k_{\text {min }}$ is given by $\gamma$ as well as $\theta$ irrespective of m.m. and that $\gamma$ and consequently $P_{m}(m=1, \ldots, n)$ are unchanged under m.m. in our artificial data. The differences of $k_{\text {min }}$ over sample sizes are due to the differences of seeds in random numbers used for generating artificial $\gamma$ 's. It is found in the

Table 1 . Values of $k_{\text {min }}$ minimizing the asymptotic mean square error for the 3PLM.

\begin{tabular}{rrrrrrrr}
\hline \multicolumn{1}{c}{$n$} & \multicolumn{1}{c}{$\theta$} \\
\hline 50 & 4.56 & 4.80 & 13.32 & 143.14 & 8.44 & 4.40 & 3.28 \\
100 & 4.28 & 4.80 & 11.18 & 792.84 & 15.06 & 4.32 & 2.97 \\
300 & 4.30 & 4.90 & 8.80 & 83.48 & 9.84 & 4.15 & 3.16 \\
\hline
\end{tabular}


table that most of the values are larger than 3 with an exceptional value of 2.97 when $\theta=3$ and $n=100$. From the table, the lower bound 3 derived under the LME is found to be reasonable even in the 3PLM as long as data are similar to those in this table. When $\theta=0$, values of $k_{\min }$ are rather large. This is expected and is explained by the small values of the denominator $\bar{j}_{0}$ in two terms of (3.9): $\bar{j}_{0}=n^{-1} \sum_{m=1}^{n} P_{m}^{\prime} P_{m}^{\prime \prime} /\left(P_{m} Q_{m}\right)$. Define $\Psi_{m} \equiv 1 /\left[1+\exp \left\{-D a_{m}\left(\theta-b_{m}\right)\right]\right.$ $(m=1, \ldots, n)$. Then,

$$
\bar{j}_{0}=n^{-1} D^{3} \sum_{m=1}^{n} a_{m}^{3}\left(1-c_{m}\right)^{2} \Psi_{m}^{2}\left(1-\Psi_{m}\right)^{2}\left(1-2 \Psi_{m}\right) /\left(P_{m} Q_{m}\right),
$$

which is 0 when $\Psi_{m}=0.5(m=1, \ldots, n)$. Though $\bar{j}_{0}=0$ is excluded by assumption, this can happen in the $3 \mathrm{PLM}$ when $b_{m}=b(m=1, \ldots, n)$ and when $\theta_{0}=b$. Even when $b_{m}$ are different, a similar result is given when $\theta_{0}$ is close to the mean of $b_{m}(m=1, \ldots, n)$, which is typically around 0 in practice and in our data.

Tables 2 to 5 give theoretical or asymptotic values i.e., the asymptotic standard error $\left(\mathrm{ASE}=n^{-1 / 2} \alpha_{\mathrm{ML} 2}^{1 / 2}\right)$, the higher-order asymptotic standard error $\left(\mathrm{HASE}=\left(n^{-1} \alpha_{\mathrm{ML} 2}+n^{-2} \alpha_{\mathrm{GW} \Delta 2}\right)^{1 / 2}\right), \alpha_{\mathrm{GW} \Delta 2}, \alpha_{\mathrm{GW} 1}^{2}$ and the higherorder asymptotic root mean square error (HRMSE $=\left\{n^{-1} \alpha_{\mathrm{ML} 2}+n^{-2}\left(\alpha_{\mathrm{GW} \Delta 2}+\right.\right.$ $\left.\left.\left.\alpha_{\mathrm{GW} 1}^{2}\right)\right\}^{1 / 2}=\left(\mathrm{HASE}^{2}+n^{-2} \alpha_{\mathrm{GW} 1}^{2}\right)^{1 / 2}\right)$ and the corresponding simulated values. Tables 2 and $3(n=50)$ and Table $4(n=100)$ are given under c.m.s. while Table $5(n=100)$ is given under m.m. The asymptotic values under m.m. are given by $P_{\mathrm{T} m}$ as well as $P_{m}(m=1, \ldots, n)$. In Table 2 , some values of HASE and HRMSE become imaginary when a relatively large $k_{\min }$ is used, where $\alpha_{\mathrm{GW} \Delta 2}$ is negative with a large absolute value. The ASE is common to all methods ML through $\mathrm{WL}\left(k_{\min }\right)$.

Simulated values corresponding to the asymptotic ones are given by simulations in the following way. Random responses by simulated examinees are generated under c.m.s. (Tables 2 to 4 ) and under m.m. (Table 5). Estimates of abilities by $\mathrm{ML}$ to $\mathrm{WL}\left(k_{\min }\right)$ are given by the generated responses, where observations with at least 1 non-convergent estimate in each set of the estimates are discarded. This was replicated until $10^{5}$ regular observations are obtained. The nonzero numbers of deleted observations are 469 when $\theta=-1$ in Table 1, 27 when $\theta=2$ in Table 3,41 when $\theta=-1$ in Table 4 and 18 when $\theta=-1$ in Table 5 . The 27 cases when $\theta=2$ in Table 3 are all due to the perfect score, where finite $\hat{\theta}_{\mathrm{ML}}$ is not available. The other deleted cases are due to non-convergence in estimation. The simulated values (denoted by SDs) common to the corresponding ASE and HASE are the square roots of the unbiased sample variances given by $10^{5}$ estimates for each estimator. The simulated $\alpha_{\mathrm{GW} \Delta 2}$ is $n^{2}\left(\mathrm{SD}^{2}-n^{-1} \alpha_{\mathrm{ML} 2}\right)$. The simulated $\alpha_{\mathrm{GW} 1}^{2}$ is the square of the simulated $\alpha_{\mathrm{GW} 1}$, which is given by $n$ times the mean of $10^{5}$ values of $\hat{\theta}_{\mathrm{GW}}-\theta_{0}$. The simulated HRMSE is given by the square root of the mean of $\left(\hat{\theta}_{\mathrm{GW}}-\theta_{0}\right)^{2}$ over $10^{5}$ replications. Note that the value of $\alpha_{\text {GW1 }}^{2}$ denoted by " 0 " for WL in Tables 2 to 4 are algebraically 0 by construction. The other ".0" s are rounded values up to the first decimal place. 


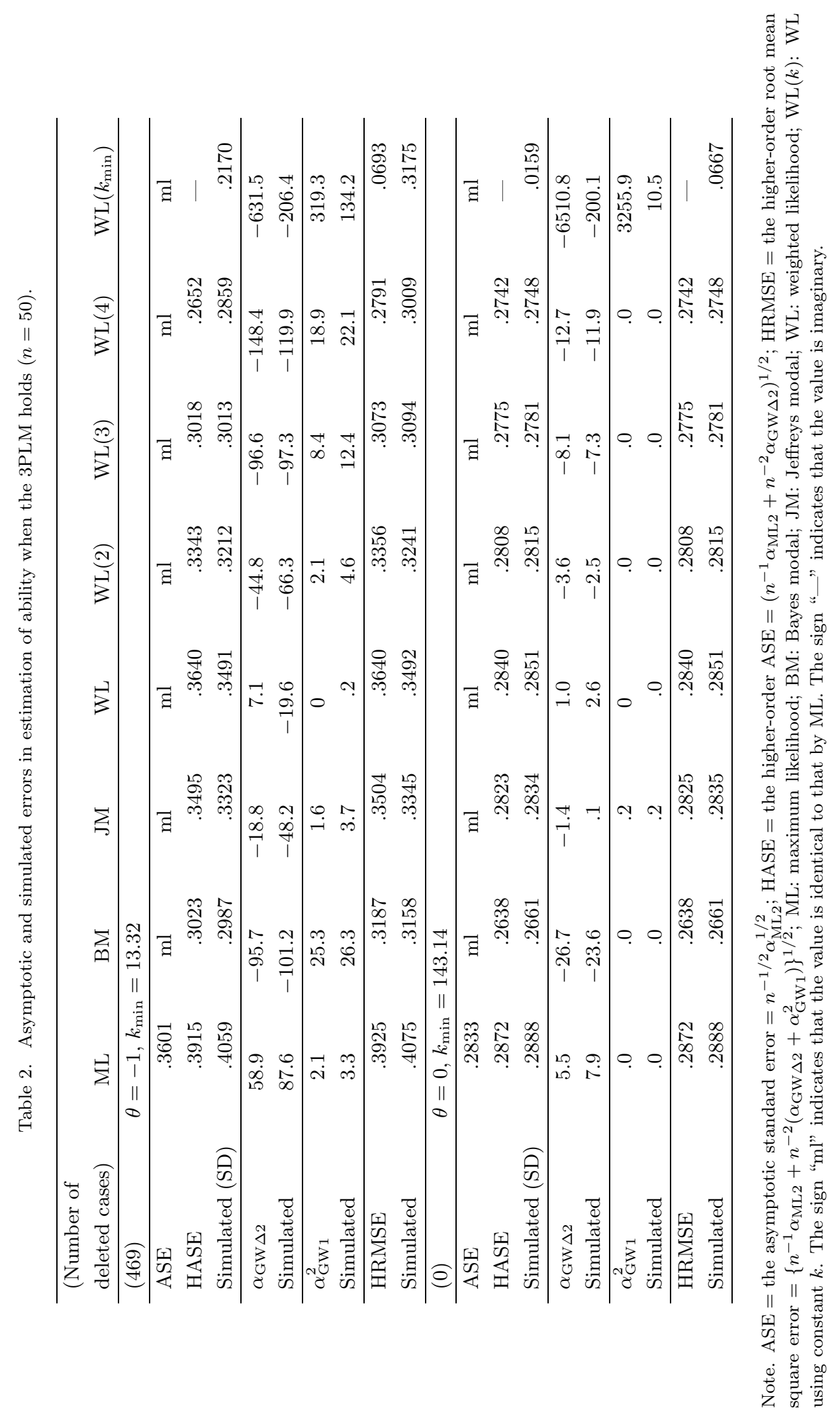




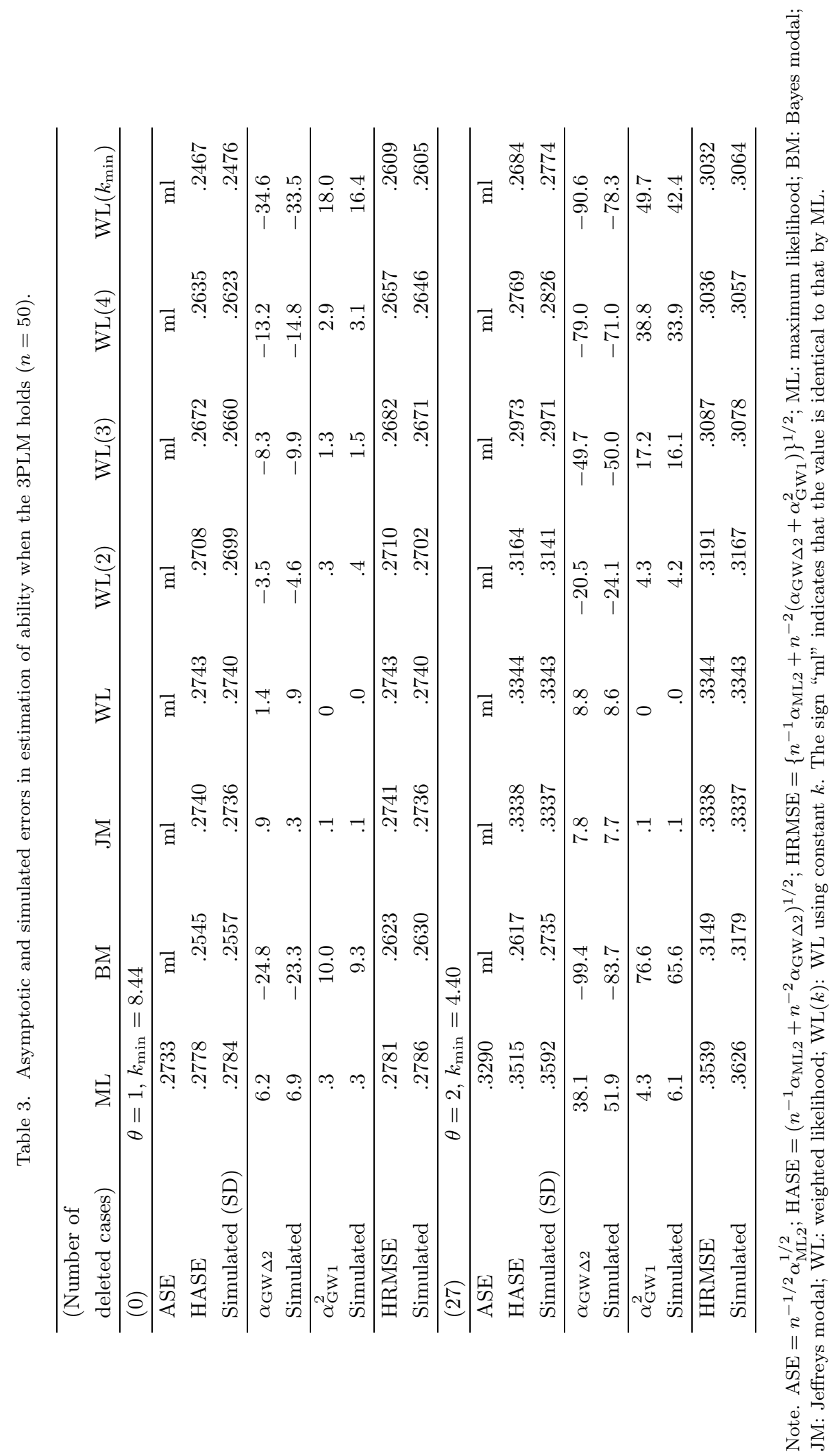




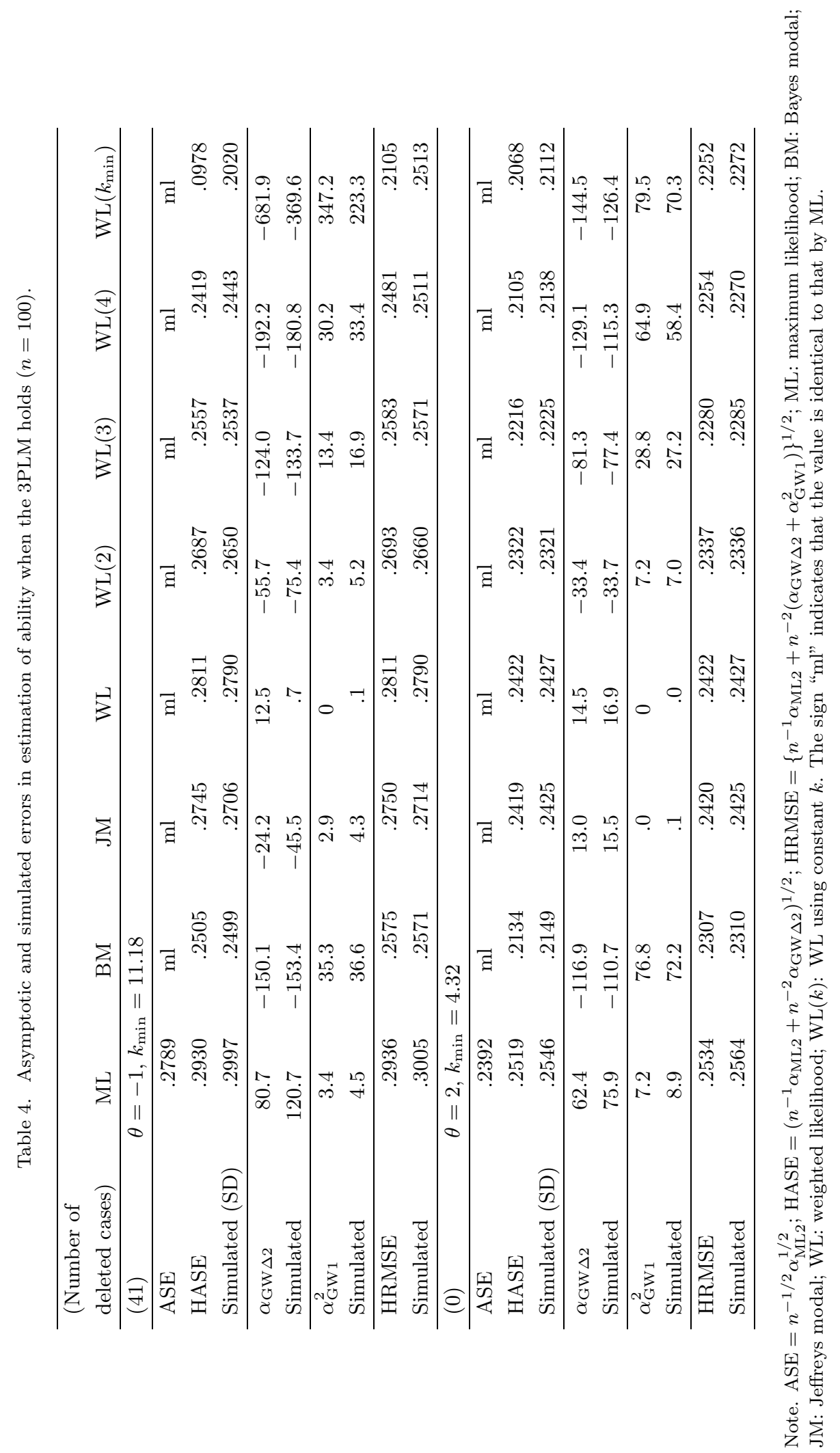




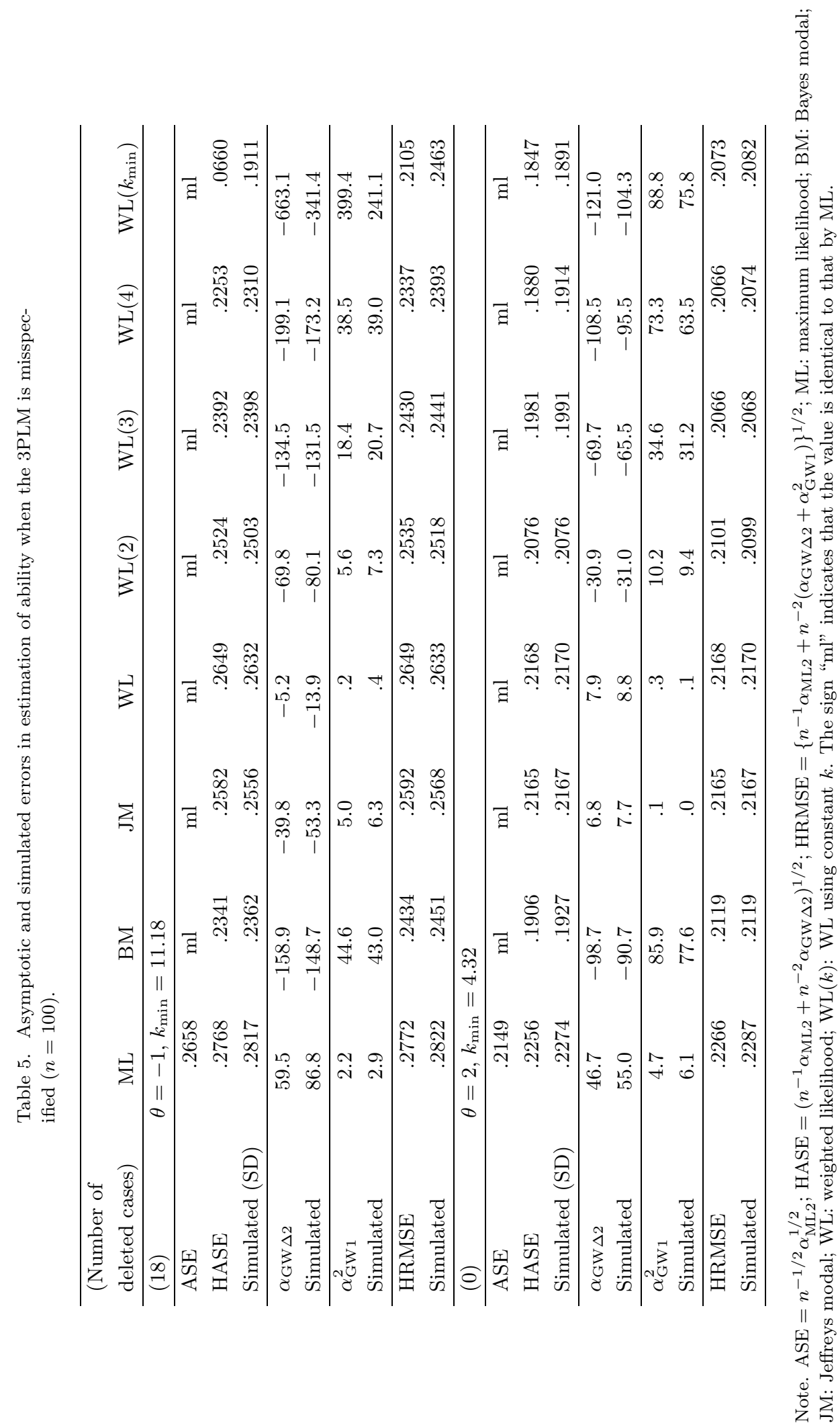


Throughout the tables, it is found that the simulated values are reasonably similar to the corresponding asymptotic values with some exceptions for $\mathrm{WL}\left(k_{\min }\right)$ when $k_{\min }$ is rather large. The $\mathrm{SD}$ values are more similar to the corresponding values of HASE than the common ASE. In Tables 2 to 4 , as mentioned earlier, the excellent property of WL is shown in that not only $\alpha_{\mathrm{GW} 1}^{2}$ is zero with similar simulated values, but also $\alpha_{\mathrm{GW} \Delta 2}$ is smaller than that of ML. For $\mathrm{WL}(k)$, the methods $\mathrm{WL}(2), \mathrm{WL}(3)$ and $\mathrm{WL}(4)$ give smaller HRMSEs with similar simulated values than those by WL. Note that these small values, especially in $\mathrm{WL}(2)$ and $\mathrm{WL}(3)$, are obtained without increasing $\alpha_{\mathrm{GW} 1}^{2}$ to a large extent as in BM. It is of interest to see that $\mathrm{WL}(4)$ gives similar results to those by BM in a crude sense. The results of JM and WL are similar, which is expected since in the 2PLM, JM and WL are identical. However, the tables show that JM gives smaller $\alpha_{\mathrm{GW} \Delta 2}$ and HRMSE than those by WL with similar simulated tendencies.

Table 5 under m.m. gives a realistic picture for estimating abilities in that the familiar 3PLM is more or less misspecified in real situations even when the calibration sample for item parameters is large enough to satisfy $N=O\left(n^{5 / 2}\right)$. The ASEs, HASEs and SDs in Table 5 are found to be substantially smaller than those in Table 2, which can be explained by a regression effect. It is again of interest to see that the results of BM and WL(4) are similar though WL(4) gives slightly smaller HRMSE than $\mathrm{BM}$ and even $\mathrm{WL}\left(k_{\min }\right)$. The last reversed result for $\mathrm{WL}(4)$ and $\mathrm{WL}\left(k_{\min }\right)$ is due to $\mathrm{m} . \mathrm{m}$. It is to be noted that the asymptotic and simulated $\alpha_{\mathrm{GW} 1}^{2}$ by WL are close to 0 irrespective of m.m.

\section{Remarks}

A natural question arises. Which method is to be chosen? This depends on how the risk of an estimator is defined and chosen. When only reduction of bias is of interest, WL should be chosen even when m.m. is expected as long as the m.m. is similar to that in Table 5. This choice is reasonable in that the variance also becomes smaller than that by ML. Since MSE is an overall index of bias and variance, the criterion of HRMSE is in many practical cases better than $n^{-1} \alpha_{\mathrm{GW} 1}$ alone. Note that the shrinkage estimators reduce variance explicitly (see e.g., Gruber (1998), Chapter 1; Lehmann and Casella (1998), Chapter 5, Section 5). Ogasawara (2013a, p. 371) recommends $\hat{\theta}_{\mathrm{BM}}$ among the estimators by ML, BM, LM and WL. The familiar estimator $\hat{\theta}_{\mathrm{BM}}$ is reasonable in that its HRMSE tends to be smaller than that obtained by WL. The corresponding counterpart of $\mathrm{WL}(k)$ is $\mathrm{WL}(4)$ as mentioned earlier. When relatively small bias is desired, $\mathrm{WL}(2)$ and $\mathrm{WL}(3)$ may be good choices. In the tables it is seen that, $\mathrm{WL}(2)$ gives $\alpha_{\mathrm{GW} 1}^{2}$ similar to that by ML, smaller $\alpha_{\mathrm{GW} \Delta 2}$, and consequently smaller HRMSE than those by ML.

The redefined weight $g(\theta)$ for $\mathrm{WL}(2)$ can be interpreted as an approximation to that of the informative logistic prior, whose density is given by $D P^{*} Q^{*}$ with $P^{*} \equiv 1 /\{1+\exp (-D \theta)\}$ and $Q^{*} \equiv 1-P^{*}$. It is known that the difference between $P^{*}$ and the cumulative distribution function of the standard normal is 
less than 0.01 for all $\theta$ (e.g., Lord and Novick (1968), Inequality (17.2.2)). The weight for the logistic prior becomes

$$
g(\theta)=\frac{\partial \log \left(D P^{*} Q^{*}\right)}{\partial \theta}=D\left(1-2 P^{*}\right)
$$

In the case of the LME, the weight for WL(2) is $2 g(\theta)=2 \bar{j} /(2 \bar{i})=\operatorname{Da}(1-2 P)$ (see (3.4)), which becomes equal to (5.1) when $a=1$ and $P=P^{*}$. Note that (5.1) corresponds to $g(\theta)=-\theta$ for $\mathrm{BM}$, where the range of $(5.1)$ is $(-D, D)$ while that of $\mathrm{BM}$ is $(-\infty, \infty)$; both of $(5.1)$ for $\mathrm{WL}(2)$ and $-\theta$ for BM are strictly decreasing functions of $\theta$; and they are simultaneously zero at $\theta=0$. The similarity of the results by $\mathrm{BM}$ to those by $\mathrm{WL}(4)$ rather than by $\mathrm{WL}(2)$ in the tables is primarily due to the 3PLM with different parameters over items as well as the difference of the logistic and normal distributions.

As mentioned in Section 3, $k g(\theta)$ can also be considered for BM, which gives $\hat{\theta}_{\mathrm{BM}(k)}$ when $k g(\theta)=-k \theta$. Since under c.m.s.,

$$
\begin{aligned}
\operatorname{MSE}_{O\left(n^{-2}\right)}\left(\hat{\theta}_{\mathrm{BM}(k)}\right) & \\
= & n^{-1} \bar{i}_{0}^{-1} \\
& \quad+n^{-2}\left[\alpha_{\mathrm{ML} \Delta 2}+\alpha_{\mathrm{ML} 1}^{2}+k\left\{-2 \bar{i}_{0}^{-2}+\bar{i}_{0}^{-3}\left(2 \bar{i}_{0}^{\prime}+\bar{j}_{0}\right) \theta_{0}\right\}+k^{2} \bar{i}_{0}^{-2} \theta_{0}^{2}\right]
\end{aligned}
$$

$(\operatorname{see}(3.1)), k_{\min }$ becomes

$$
k_{\min }=\theta_{0}^{-2}-\bar{i}_{0}^{-1}\left(\bar{i}_{0}^{\prime}+\frac{\bar{j}_{0}}{2}\right) \theta_{0}^{-1} .
$$

When the 2PLM holds, (5.3) becomes

$$
k_{\min }=\theta_{0}^{-2}-\frac{3}{2} \bar{i}_{0}^{-1} \bar{j}_{0} \theta_{0}^{-1} .
$$

The second term on the right-hand side of (5.4) tends to be positive because $\bar{j}_{0}=\bar{i}_{0}^{\prime}$ tends to be positive (negative) when $\theta_{0}$ is negative (positive). Though it is not easy to find a fixed positive lower bound which does not depends on $\theta_{0}$ in $\operatorname{BM}(k)$, probably $\mathrm{BM}=\mathrm{BM}(1)$ is reasonable enough since in the tables $\mathrm{BM}$ gives results similar to those by $\mathrm{WL}(4) . \mathrm{BM}(k)$ with $k<1$ is expected to yield results similar to those by $\mathrm{WL}(k)$ with $k<4$.

As mentioned earlier, the value $k$ in $\operatorname{BM}(k)$ is interpreted as the reciprocal of the variance in the normal prior. While the normal prior is an informative one based on the subjective probability of $\theta_{0}$ from the Bayesian viewpoint, the prior is reasonable in that calibration of item parameters is in many cases performed such that $\theta$ s are integrated out using the standard normal for the distribution of $\theta$.

So far, in this paper $k g(\theta)$ is used to have $\hat{\theta}_{\mathrm{GW}(k)}$. However, an asymptotically similar estimator corresponding to $\hat{\theta}_{\mathrm{GW}(k)}$ is obtained after $\hat{\theta}_{\mathrm{ML}}$ is given, followed by correction yielding $\hat{\theta}_{\mathrm{C}-\mathrm{GW}(k)}$ :

$$
\hat{\theta}_{\mathrm{C}-\mathrm{GW}(k)} \equiv \hat{\theta}_{\mathrm{ML}}+n^{-1} k \hat{\alpha}_{\mathrm{ML} 2} g\left(\hat{\theta}_{\mathrm{ML}}\right) .
$$


A familiar $\hat{\theta}_{\mathrm{C}-\mathrm{GW}(k)}$ is $\hat{\theta}_{\mathrm{C}-\mathrm{WL}(1)}=\hat{\theta}_{\mathrm{C}-\mathrm{WL}}=\hat{\theta}_{\mathrm{ML}}-n^{-1} \hat{\alpha}_{\mathrm{ML} 1}$, which is an asymptotically bias-corrected MLE, where $\hat{\alpha}_{\mathrm{ML} 1}$ is the sample counterpart of $\alpha_{\mathrm{ML} 1}$. Recall that in $\hat{\theta}_{\mathrm{WL}}, g(\theta)=\bar{j} /(2 \bar{i})$, which gives $\hat{\alpha}_{\mathrm{ML} 2} g\left(\hat{\theta}_{\mathrm{ML}}\right)=\hat{\bar{i}}^{-1}\{\hat{\bar{j}} /(2 \hat{\bar{i}})\}=$ $-\left\{-\hat{\bar{j}} /\left(2 \hat{\bar{i}}^{2}\right)\right\}=-\hat{\alpha}_{\mathrm{ML} 1}$ under c.m.s. (see the first paragraph of Section 3 ), where $\hat{\bar{i}}$ and $\hat{\bar{j}}$ are sample versions of $\bar{i}$ and $\bar{j}$, respectively. Ogasawara (2013a, Theorem 4) showed that the asymptotic cumulants up to the fourth order and the higher-order asymptotic variance of $\hat{\theta}_{\mathrm{C}-\mathrm{WL}(k)}$ are identical to those of $\hat{\theta}_{\mathrm{WL}(k)}$.

The equality of $\mathrm{MSE}_{O\left(n^{-2}\right)}$ 's of $\hat{\theta}_{\mathrm{WL}(k)}$ and $\hat{\theta}_{\mathrm{C}-\mathrm{WL}(k)}$ is shown as follows. From the above result, we have $g\left(\theta_{0}\right)=\bar{j}_{0} /\left(2 \bar{i}_{0}\right)=-\bar{i}_{0} \alpha_{\text {ML1 }}$. Then, under c.m.s.,

$$
\begin{aligned}
& \operatorname{MSE}_{O\left(n^{-2}\right)}\left(\hat{\theta}_{\mathrm{WL}(k)}\right)-n^{-1} \bar{i}_{0}^{-1} \\
& =n^{-2}\left[\alpha_{\mathrm{ML} \Delta 2}+\alpha_{\mathrm{ML} 1}^{2}+k\left\{2 \bar{i}_{0}^{-2} g^{\prime}\left(\theta_{0}\right)-\bar{i}_{0}^{-3}\left(2 \bar{i}_{0}^{\prime}+\bar{j}_{0}\right) g\left(\theta_{0}\right)\right\}\right. \\
& \left.+k^{2} \bar{i}_{0}^{-2} g\left(\theta_{0}\right)^{2}\right] \\
& =n^{-2}\left[\alpha_{\mathrm{ML} \Delta 2}+\alpha_{\mathrm{ML} 1}^{2}+k\left\{-2 \bar{i}_{0}^{-2}\left(\bar{i}_{0}^{\prime} \alpha_{\mathrm{ML} 1}+\bar{i}_{0} \partial \alpha_{\mathrm{ML} 1} /\left.\partial \theta\right|_{\theta=\theta_{0}}\right)\right.\right. \\
& \left.\left.+\bar{i}_{0}^{-2}\left(2 \bar{i}_{0}^{\prime}+\bar{j}_{0}\right) \alpha_{\mathrm{ML} 1}\right\}+k^{2} \alpha_{\mathrm{ML} 1}^{2}\right] \\
& =n^{-2}\left\{\alpha_{\mathrm{ML} \Delta 2}+\alpha_{\mathrm{ML} 1}^{2}-2 k\left(\bar{i}_{0}^{-1} \partial \alpha_{\mathrm{ML} 1} /\left.\partial \theta\right|_{\theta=\theta_{0}}+\alpha_{\mathrm{ML} 1}^{2}\right)+k^{2} \alpha_{\mathrm{ML} 1}^{2}\right\} \\
& =n^{-2}\left[\alpha_{\mathrm{ML} \Delta 2}+\alpha_{\mathrm{ML} 1}^{2}-2 k\left\{n \operatorname{acov}\left(\hat{\theta}_{\mathrm{ML}}, \hat{\alpha}_{\mathrm{ML} 1}\right)+\alpha_{\mathrm{ML} 1}^{2}\right\}+k^{2} \alpha_{\mathrm{ML} 1}^{2}\right] \\
& =n^{-2}\left\{\alpha_{\mathrm{ML} \Delta 2}+(1-k)^{2} \alpha_{\mathrm{ML} 1}^{2}-2 k n \operatorname{acov}\left(\hat{\theta}_{\mathrm{ML}}, \hat{\alpha}_{\mathrm{ML} 1}\right)\right\} \\
& =\operatorname{MSE}_{O\left(n^{-2}\right)}\left(\hat{\theta}_{\mathrm{C}-\mathrm{WL}(k)}\right)-n^{-1} \bar{i}_{0}^{-1},
\end{aligned}
$$

where $\operatorname{acov}(\cdot, \cdot)$ is the asymptotic covariance of order $O\left(n^{-1}\right)$ for the two variables in parentheses. Note that in $\hat{\theta}_{\mathrm{C}-\mathrm{WL}(k)}=\hat{\theta}_{\mathrm{ML}}-n^{-1} k \hat{\alpha}_{\mathrm{ML} 1}, E\left(\hat{\theta}_{\mathrm{C}-\mathrm{WL}(k)}\right)=n^{-1}(1-$ k) $\alpha_{\mathrm{ML} 1}+O\left(n^{-2}\right)$ and $\operatorname{var}\left(n^{-1} k \hat{\alpha}_{\mathrm{ML} 1}\right)=O\left(n^{-3}\right)$, where the last variance can be neglected for evaluation of $\operatorname{MSE}_{O\left(n^{-2}\right)}\left(\hat{\theta}_{\mathrm{C}-\mathrm{WL}(k)}\right)$. Since in Ogasawara (2013a, Theorem 4$), g(\cdot)$ can be other than that of $\hat{\theta}_{\mathrm{WL}(k)}$, similar results are obtained for $\hat{\theta}_{\mathrm{C}-\mathrm{GW}(k)}$ with general $g(\cdot)$.

In the case of estimation of ability, finite $\hat{\theta}_{\mathrm{ML}}$ is not available for the zero and perfect scores, which gives an advantage of $\hat{\theta}_{\mathrm{GW}(k)}$ over $\hat{\theta}_{\mathrm{C}-\mathrm{GW}(k)}$. The latter corrected estimator may have an advantage in that the computer program tends to be simple since an iterative computation is required only for $\hat{\theta}_{\mathrm{ML}}$.

\section{Acknowledgements}

This work was partially supported by a Grant-in-Aid for Scientific Research from the Japanese Ministry of Education, Culture, Sports, Science and Technology, No. 23500341.

\section{REFERENCES}

Birnbaum, A. (1968). Some latent trait models and their use in inferring an examinee's ability, Statistical Theories of Mental Test Scores (ed. F. M. Lord and M. R. Novick), Reading, MA, Addison-Wesley.

Birnbaum, A. (1969). Statistical theory for logistic mental test models with a prior distribution of ability, J. Math. Psychol., 6, 258-276. 
Bock, R. D. and Aitkin, M. (1981). Marginal maximum likelihood estimation of item parameters: Application of an EM algorithm, Psychometrika, 46, 443-459.

Firth, D. (1993). Bias reduction of maximum likelihood estimates, Biometrika, 80, 27-38.

Gruber, M. H. J. (1998). Improving Efficiency by Shrinkage: The James-Stein and Ridge Regression Estimators, New York, Marcel Dekker.

Jeffreys, H. (1946). An invariant form for the prior probability in estimation problems, Proc. R. Soc. Lond. Ser. A, 186, 453-461.

Jeffreys, H. (1961). Theory of Probability (3rd ed.), Oxford, Clarendon.

Kolen, M. J. and Brennan, R. L. (2004) Test Equating, Scaling, and Linking: Methods and Practices (2nd ed.), New York, Springer.

Lehmann, E. L. and Casella, G. (1998). Theory of Point Estimation (2nd ed.), New York, Springer.

Lord, F. M. (1953). An application of confidence intervals and of maximum likelihood to the estimation of an examinee's ability, Psychometrika, 18, 57-76.

Lord, F. M. (1975). Evaluation with Artificial Data of a Procedure for Estimating Ability and Item Characteristic Curve Parameters (Research Bulletin 75-33), Princeton, NJ, Educational Testing Service.

Lord, F. M. (1983). Unbiased estimators of ability parameters, of their variance, and of their parallel-forms reliability, Psychometrika, 48, 233-245.

Lord, F. M. and Novick, M. R. (1968). Statistical Theories of Mental Test Scores, Reading, MA, Addison-Wesley.

Ogasawara, H. (2012). Asymptotic expansions for the ability estimator in item response theory, Comp. Stat., 27, 661-683.

Ogasawara, H. (2013a). Asymptotic properties of the Bayes and pseudo Bayes estimators of ability in item response theory, J. Multivar. Anal., 114, 359-377.

Ogasawara, H. (2013b). Bias adjustment minimizing the asymptotic mean square error, Commun. Stat., Theory and Methods, DOI:10.1080/03610926.2013.786788.

Ogasawara, H. (2013c). Asymptotic cumulants of the ability estimators using fallible item parameters, J. Multivar. Anal., 119, 144-162.

Samejima, F. (1969). Estimation of latent ability using a response pattern of graded scores, Psychometrika Monograph, No. 17.

Warm, T. A. (1989). Weighted likelihood estimation of ability in item response theory, Psychometrika, 54, 427-450. 